\title{
The potential of ciRS-7 for predicting onset and prognosis of cervical cancer
}

\author{
Y. ZHOU*, L. SHEN, Y. Z. WANG, C. C. ZHOU \\ Department of Obstetrics and Gynecology, Xinhua Hospital Affiliated to Shanghai Jiaotong University School of Medicine, Shanghai, China \\ *Correspondence: zhouyunyun08@126.com
}

Received April 15, 2019 / Accepted July 24, 2019

\begin{abstract}
Considering the potency of circRNAs in manifesting neoplastic progression, we attempted to explore the feasibility of applying ciRS-7 for diagnosis and prognosis estimation of cervical cancer (CC). Here 352 CC patients, 204 cervical intraepithelial neoplasia (CIN) patients and 227 healthy controls were recruited. The Kaplan-Meier survival curves were fitted to estimate associations of ciRS-7 expression with CC prognosis, and we also adopted receiver operating characteristic (ROC) curves to assess the diagnostic performance of ciRS-7 for CC. Meanwhile, endometrial stromal cell line (ESC) and 4 human CC cell lines (i.e. Hela, CaSki, C33A and SiHa) were also gathered. After transfection of pcDNA3.1-ciRS-7, impacts of overexpressed ciRS-7 on proliferation, apoptosis, invasion and migration of CC cells were assessed through performing colony formation assay, flow cytometry, transwell assay and wound healing assay. The results demonstrated that CC patients that highly expressed ciRS-7 were associated with high odds of large tumor size, advanced FIGO stage, deep invasion, metastatic lymph nodes and HPV infection $(\mathrm{p}<0.05)$. Furthermore, ciRS-7 excelled in differentiating CC patients from healthy controls and CIN patients than CEA and CA125 ( $p<0.05$ ), and ciRS-7 combined with CA125 and CEA generated an optimum efficacy in diagnosing CC patients and CIN patients from healthy controls $(\mathrm{p}<0.05)$. Concerning in vitro experiments, elevating ciRS-7 expression significantly intensified proliferation and epithelial-mesenchymal transition (EMT) of CC cells $(p<0.05)$, and also markedly suppressed apoptosis of the cells $(p<0.05)$. In conclusion, ciRS-7 possessed great potential in clinical diagnosis of CC, given its involvement in modulating the activity of CC cells.
\end{abstract}

Key words: ciRS-7, cervical cancer, CEA, CA125, combined diagnosis, ROC curve, cell proliferation, epithelial-mesenchymal transition

Cervical cancer (CC), one commonly detectable gynecological cancer, stood merely next to breast cancer in terms of incidence among diversified neoplasms [1]. One pivotal contributor to incremental CC risk was proposed as persistent infection of high-risk human papillomavirus (HR-HPV), and the age of CC onset assumed a younger trend due to increasingly prominent environmental pollution, heavy work pressure and poor hygiene [2]. Moreover, although CC prognosis has been largely ameliorated after introduction of surgery and radiotherapy, it remained challenging to radically cure metastatic CC and prevent CC recurrence. Of note, the occurrence of $\mathrm{CC}$ was documented as a result of malignant changes and infinite multiplication of cervical epithelial cells, which was accompanied by functional changes of multiple genes (e.g. p53, Bcl-2 and c-myc) [3-5]. From the above, it seemed that discovery of appropriate biomarkers that indicated pathogenesis underlying CC metastasis might conduce to effective treatment of CC.

The circular RNAs (circRNAs), since the discovery by Sanger et al. and Kolakofsky et al. in 1979, have been increas- ingly valued regarding their implications in modulating neoplastic progression $[6,7]$. To be specific, gastric cancer patients with lymphatic metastases were examined with reduced expression of hsa_circ_002058 [8], and hsa circ_100855 seemed as a protective molecule against neurotropic invasion of colorectal cancer [9]. By contrast, cerebellar degeneration-associated protein 1 antisense transcript (ciRS-7) was confirmed as a facilitator for progression of neoplasms, including hepatocellular carcinoma [10], gastric cancer [11] and esophageal squamous cell carcinoma [12]. Despite the absence of direct evidences, ciRS- 7 could also be accountable for deterioration of CC owing to its possession of miRNA response elements (MREs) that were specific to miR-7 [13], whose expressional alteration could be representative of CC progression [14]. As previously documented, circRNAs could interplay with miRNAs and thereby blocked or promoted expression of tumor-characteristic genes. For instance, circ-ZEB1.33 sponging miR-200a-3p was found to strengthen the proliferative capability of hepatocellular carcinoma cells by raising expression of CDK6 [15]. Concerning 
ciRS-7 investigated here, its binding to miR-7 also could trigger expressional changes of molecules that displayed relevance to neoplastic progression, such as insulin-like growth factor-1 receptor (IGF1R), epidermal growth factor receptor (EGFR) and focal adhesion kinase (FAK) [16]. Virtually, the miR-7 could also exert inhibitory impacts on the aggravation of cancers besides CC, such as breast cancer and lung cancer $[17,18]$. To sum up, it seemed probable that ciRS-7 could matter in tumorigenesis (e.g. CC) via acting on miR-7.

Nonetheless, this point remained unclear so far, due to shortage of relevant clinical and in vitro evidences. To explore this untapped field, we attempted to find out whether ciRS-7 could be a reliable biomarker for diagnosing CC, and through which molecular mechanism ciRS-7 functioned to modulate CC progression.

\section{Materials and methods}

Inclusion of CC clinical samples. From July 2015 to November 2017, total of 352 pairs of CC tissues and adjacent non-tumor tissues were gathered from the primary CC patients who received surgeries in the obstetrics and gynecology department of Xinhua Hospital Affiliated to Shanghai Jiaotong University School of Medicine. The included CC patients were all pathologically confirmed with CC, and their severities were classified according to the criteria formulated by International Federation of Obstetrics and Gynecology (FIGO). Moreover, the CC cases underwent none of radiotherapy, chemotherapy and hormone therapy before the surgery, and those with serious infectious diseases, hepato-renal insufficiency, non-neoplastic lesions and other tumors were excluded. Meanwhile, we also recruited 204 cervical intraepithelial neoplasia (CIN) patients and 227 healthy females who performed physical examinations here. Finally, this investigation was approved by Xinhua Hospital Affiliated to Shanghai Jiaotong University School of Medicine and the ethics committee of Xinhua Hospital Affiliated to Shanghai Jiaotong University School of Medicine. All the participants or their direct relatives have signed informed consents. Of note, whether to participate in this investigation was fully dependent on the willingness of participants themselves, and only when the participants agreed to take part in this program could the consents be signed by their direct relatives.

Detection of carcino-embryonic antigen (CEA) and carbohydrate antigen (CA)-135. In the morning, around 3-5 ml venous blood was withdrawn from each fasting participant. After centrifuging the blood at $3000 \mathrm{rpm}$ for 10 min, the resultant serum was arranged for detection of CEA and CA-135 on the instrument for electrochemiluminescence analysis (model: E170, Roche, Sweden). The subjects would be classified as CC-positive when their CEA level was $>5 \mathrm{mg} / \mathrm{ml}$ or when their CA-125 level was $>35 \mathrm{U} / \mathrm{ml}$. All experiments were repeated for $\geq 3$ times.
Cell culture and cell transfection. The human CC cell lines (i.e. Hela, CaSki, C33A and SiHa cell lines) were purchased from American Type Culture Collection (ATCC) (USA), and the endometrial stromal cell line (ESC) was provided by the Cell Biology Institute of Chinese Academy of Science (Shanghai, China). The cell lines were cultivated within RPMI 1640 supplemented with 10\% fetal bovine serum (FBS), and the cultivation atmosphere was maintained as $37^{\circ} \mathrm{C}$ and $5 \% \mathrm{CO}_{2}$. Moreover, pcDNA3.1-ciRS-7 and pcDNA3.1 (Genepharma, China) were transfected into Hela and C33A cell lines, according to the instructions of Lipofectamine $^{\text {rm }} 2000$ kit (Invitrogen, USA). Forty-eight hours later, the cells were digested with trypsin for subsequent cellular experiments. The above-mentioned experiments were repeated for $\geq 3$ times.

Real time-polymerase chain reaction (RT-PCR). The total RNA was extracted from tissues with usage of TRIzol reagent (Invitrogen, USA), and its concentration and purity were evaluated usage of ultra-violet detector (Bio-rad, USA). The purity of total RNA was guaranteed to be within the range of 1.6-1.8. Subsequently, the total RNA was reversely transcribed into cDNAs, according to the instructions from PrimeScript ${ }^{\mathrm{m}} \mathrm{RT}$ Master Mix kit (TaKaRa, Japan). After that, PCR was performed with assistance of real-time SYBR Premix-Ex-Taq ${ }^{\text {tix }}$ II kit (TaKaRa, Japan), and the specified procedures included pre-degeneration at $95^{\circ} \mathrm{C}$ for $30 \mathrm{~s}$, as well as 40 cycles of $95^{\circ} \mathrm{C}$ for $5 \mathrm{~s}$ and $60^{\circ} \mathrm{C}$ for $34 \mathrm{~s}$. Moreover, the primers for ciRS-7 (sense: 5'-ACGTCTCCAGTGTGCTGA-3', anti-sense: 5'-CTTGACACAGGTGCCATC-3') and GAPGH (sense: 5'-ACGTCTCCAGTGTGCTGA-3', anti-sense: 5'-CTTGACACAGGTGCCATC-3') were synthesized by Sangon Biotechnology (Shanghai, China). The expression of for ciRS-7 was quantified by $2^{-\Delta \Delta C T}$ method [12], with GAPGH as the internal reference. All these experiments conducted were repeated for $\geq 3$ times.

Western blotting. The total proteins were extracted from cells via addition of $20 \mu$ l RIPA lysis buffer (Beyotime, China), and their concentration was determined following BCA method. The proteins were separated through $10 \%$ sodium dodecyl sulfate polyacrylamide gel electrophoresis (SDS-PAGE) (Bio-RAD, USA), and the resultants were then transferred onto the polyvinylidene fluoride (PVDF) membrane (Millipore, USA) via a wet method. After $2 \mathrm{~h}$ blockage with $5 \%$ skimmed milk at room temperature, the rabbit-anti-human primary antibodies (Abcam, USA) against Ki-67 (1:5000, Cat. No.: ab92742), PCNA (1:1000, Cat. No.: ab92552), cyclinD1 (1:200, Cat. No.: ab16663), E-cadherin (1:500, Cat. No.: ab15148), N-cadherin (1:1000, Cat. No.: ab76057), Vimentin (1:2000, Cat. No.: ab137321) and GAPDH (1:2500, Cat. No.: ab9485) were used for overnight incubation at $4^{\circ} \mathrm{C}$. After washing cells with TBST for 3 times, the goat-anti-rabbit secondary antibodies labeled by horse radish peroxidase (HRP) (1:5000, Cat. No.: ab97080, Abcam, USA) were added for another $1.5 \mathrm{~h}$ incubation at room temperature. Finally, electro-chemiluminescence was 
applied for development, and the experimental results were analyzed by virtue of ImageJ software (National Institutes of Health, USA). All experiments were repeated for $\geq 3$ times.

Transwell assay. At first, the transwell chambers (Corning, USA) were placed into 24 -well plates, and the membrane in the bottom was coated and hydrated with diluent of Matrigel. Then $200 \mu \mathrm{l}$ cell suspension $\left(5 \times 10^{5} \mathrm{cells} / \mathrm{ml}\right)$ was added to the upper chamber, and $500 \mu \mathrm{l}$ DMEM medium supplemented with $20 \%$ FBS was poured into the lower chamber. After 12 h cell cultivation, the transwell chambers were taken out, and cells on the upper chamber were gently wiped out. Then the membranes were fixed with $4 \%$ methanol for $15 \mathrm{~min}$, and cotton swabs were employed to erase cells in the upper chamber. After the fixation procedure, $0.1 \%$ crystal violet was used to stain cells for $30 \mathrm{~min}$ at room temperature. Finally, 5 views were randomly selected under the microscope to count the number of invasive cells. Concerning the migration assay, most of its steps were identical to the invasion assay, except that the Matrigel was not used. All the above-mentioned experiments were repeated for $\geq 3$ times.

Wound healing assay. The cells at the density of $5 \times 10^{4} /$ well were inoculated into 6-well plates. On the next day, a straight line was drawn in the bottom of 6-well plates via the tip of a micro-syringe. After being rinsed with PBS, the cells were cultivated within serum-free medium for $48 \mathrm{~h}$. Pictures were taken at $0 \mathrm{~h}$ and $48 \mathrm{~h}$ time points, and the healing area was equivalent to the difference between areas of $0 \mathrm{~h}$ time point and $48 \mathrm{~h}$ time point. The experiments were repeated for $\geq 3$ times.

CCK-8 assay. $100 \mu \mathrm{l}$ cell suspension $\left(1 \times 10^{4}\right.$ cells $\left./ \mathrm{ml}\right)$ was added into each well along with $200 \mu \mathrm{L}$-Hanks solution for overnight cell cultivation. Then the cells were mixed with $10 \mu \mathrm{l}$ CCK8 solution (Dojindo Laboratories, Kumamoto, Japan) for another $4 \mathrm{~h}$ at $37^{\circ} \mathrm{C}$. Eventually, the optical density (OD) of each well was determined on the microplate reader at the wavelength of $450 \mathrm{~nm}$. The experiments were repeated for $\geq 3$ times.

Colony formation assay. The cells at the logarithmic phase were prepared into single-cell suspension with addition of RPMI-1640 medium that contained 10\% FBS. After being cultivated in the atmosphere of $5 \% \mathrm{CO}_{2}$ for 2 weeks, the cells were rinsed with PBS for 3 times. Subsequently, we added 5 $\mathrm{ml}$ methanol (4\%) to fix cells for $15 \mathrm{~min}$, and $500 \mu \mathrm{l}$ Giemsa liquid $(0.1 \%)$ to stain the cells for $30 \mathrm{~min}$. Ultimately, the colonies with $>50$ cells were counted under the microscope (Nikon, Japan), and the colony-formation efficiency was equivalent to the number of colonies divided by the number of inoculated cells. All experiments as above were repeated for $\geq 3$ times.

Cell apoptosis assay. The cells at the density of $1 \times 10^{6} / 1$ were centrifuged at $1200 \mathrm{xg}$ for $5 \mathrm{~min}$, and then $10 \mu \mathrm{l}$ Annexin $\mathrm{V}$-fluorescein isothiocyanate (FITC) and $5 \mu \mathrm{l}$ propidium iodide (PI) (BD, USA) were added to cultivate cells in the dark at room temperature for $15 \mathrm{~min}$. Within the bivariate scatter diagram derived from flow cytometry (model: FAC
Sort, BD, USA), the dots in the right upper quadrant (FITC+/ $\mathrm{PI}+$ ) were symbolic of late-apoptotic cells and necrotic cells. Besides, dots in the right lower quadrant (FITC+/PI-) represented cells of early-apoptotic stage, and the dots in the left lower quadrant (FITC-/PI-) symbolized viable cells. All these experiments were repeated for $\geq 3$ times.

Statistical analyses. All the statistical analyses were accomplished through usage of SPSS16.0 software. The quantitative data were compared by adoption of Student's $t$ test or analysis of variance (ANOVA) followed by Bonferroni post-hoc test. On the other hand, the enumeration data were analyzed through performing chi-square test. Moreover, Kaplan-Meier analysis was applied to devise survival curves, with Log-rank test for evaluation of significance. Besides, the Cox proportional hazard model was adopted to carry out uni-/multi-variate regression analyses. Finally, statistical distinctions were identified in case of $\mathrm{p}<0.05$.

\section{Results}

Comparison of the baseline features among CC patients, CIN patients and healthy controls. The CC patients displayed higher ciRS-7 expression than both CIN patients and healthy controls $(\mathrm{p}<0.05)$, and the ciRS-7 expression measured in CIN patients was above that detected in healthy controls $(\mathrm{p}<0.05)$ (Figure 1A). Moreover, the CEA and CA125 levels detected in the CC patients also surpassed that in the CIN patients and healthy people $(\mathrm{p}<0.05)$ (Table 1$)$. Nonetheless, hardly any significant distinctions were observed among CC patients, CIN patients and healthy controls, with regard to mean age, incidence of menopause and body mass index (BMI) (all p>0.05).

Associations of ciRS-7 expression with baseline characteristics and prognosis of CC patients. The ciRS-7 was abundantly expressed within CC tissues, when compared with adjacent non-tumor tissues (Figure 1B). Furthermore, with the median expression of ciRS-7 as the threshold, the CC patients were categorized into highly expressed $(>6.03)$ ciRS-7 group $(n=206)$ and lowly expressed $(\leq 6.03)$ ciRS-7 group $(n=146)$. It was intriguing to observe that the CC patients carrying highly expressed ciRS-7 were associated with shortened survival, when compared with those carrying lowly expressed ciRS-7 ( $\mathrm{p}<0.05)$ (Figure 1C). Besides, highly expressed ciRS-7 was more frequently detected among CC patients who were characterized by large tumor size $(\geq 4 \mathrm{~cm})$, advanced FIGO stage (II), deeper invasion $(\geq 2 / 3)$, metastatic lymph nodes and HPV infection than lowly expressed ciRS-7 $(p<0.05)$ (Table 2). The univariate analysis and multivariate analyses further demonstrated that highly expressed ciRS-7, advanced FIGO stage (II) and deep invasion $(\geq 2 / 3)$ were significant predictors for the unfavorable outcome of CC patients $(\mathrm{p}<0.05)$ (Table 3$)$.

The performance of ciRS-7, CEA and CA125 for diagnosis of CC. The ciRS-7 was outstanding in differentiating $\mathrm{CC}$ patients from healthy controls $(\mathrm{AUC}=0.972)$, when 
A

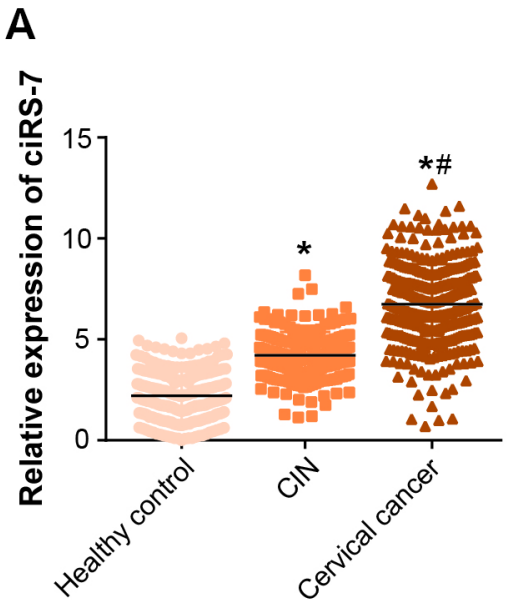

B

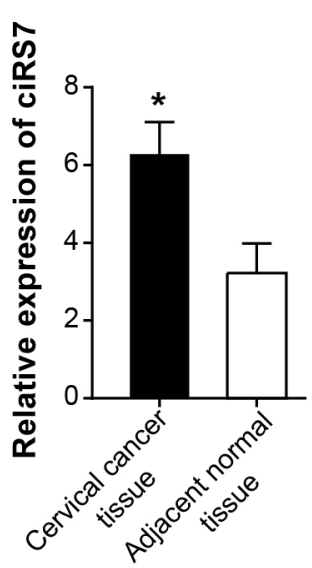

C

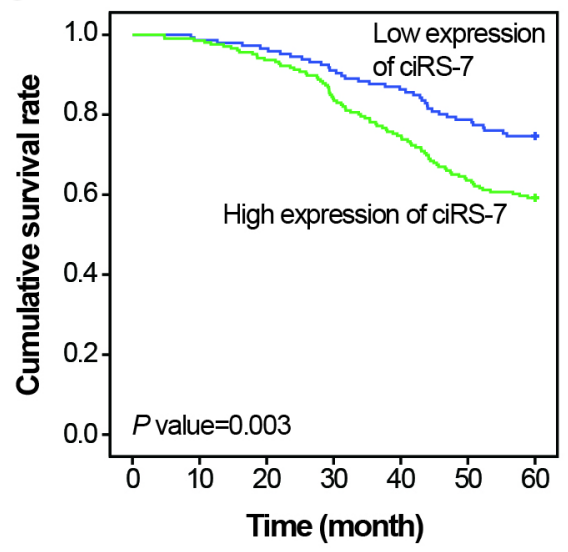

Figure 1 The expression of ciRS-7 within groups of cervical cancer patients, CIN patients and healthy controls. A) The expression of ciRS-7 was compared among cervical cancer patients, CIN patients and healthy controls. ${ }^{*}: \mathrm{p}<0.05$ when compared with healthy controls; \#: p<0.05 when compared with CIN. B) The expression of ciRS-7 was determined within cervical cancer tissues and adjacent normal tissues. ${ }^{\star}:$ p $<0.05$ when compared with adjacent normal tissues. $C)$ The upregulated ciRS-7 expression was associated with unfavorable prognosis of cervical cancer patients. ${ }^{\star}:$ p $<0.05$ when compared with healthy controls.

Table 1. Comparison of the baseline features among cervical cancer patients, cervical intraepithelial neoplasia patients and healthy controls.

\begin{tabular}{llll}
\hline Features & Healthy Control & CIN & Cervical cancer \\
\hline Number & 227 & 204 & 352 \\
Age (years old) & $52.07 \pm 9.08$ & $51.67 \pm 8.14$ & $52.84 \pm 8.75$ \\
Menopause & & & \\
$\quad$ Yes & 138 & 111 & 201 \\
$\quad$ No & 89 & 93 & 151 \\
BMI $\left(\mathrm{kg} / \mathrm{m}^{2}\right)$ & $24.18 \pm 3.81$ & $24.34 \pm 3.5$ & $24.76 \pm 4.03$ \\
CEA $(\mathrm{ng} / \mathrm{ml})$ & $2.86 \pm 1.51$ & $5.74 \pm 1.35^{*}$ & $9.06 \pm 4.7^{*}$ \\
CA125 $(\mathrm{U} / \mathrm{ml})$ & $20.19 \pm 8.42$ & $32.01 \pm 15.46^{*}$ & $48.55 \pm 22.05^{*} \#$ \\
\hline
\end{tabular}

CIN: cervical intraepithelial neoplasia. ${ }^{\star}$ Compared with healthy control, $\mathrm{p}<0.05$; \# Compared with CIN, $\mathrm{p}<0.05$.

compared with CEA (AUC=0.885) and CA125 (AUC=0.881) (Table 4, Figures 2A-2C). Apart from that, ciRS-7 stood out in diagnosing CC patients from CIN patients (AUC=0.868). In addition, CEA (AUC $=0.920$ ) could serve as a preferred biomarker in discriminating CIN population from healthy controls, as compared with ciRS-7 (AUC $=0.879)$ and CA125 (AUC=0.739).

The combined role of ciRS-7, CEA and CA125 in diagnosis of CC. Strategies of ciRS-7 combined with CEA (AUC $=0.990)$ and ciRS-7 combined with CA125 (AUC $=0.990)$ were more capable in discriminating between CC patients and healthy controls than CEA combined with CA125 (AUC=0.963) (Table 5, Figures 2A-2C). Furthermore, ciRS-7 combined with CEA (AUC=0.904) topped in differentiating CIN patients from CC patients, when compared with ciRS-7 combined with CA125 (AUC=0.895) and CEA combined with CA125 (AUC=0.814). Last but not the least, ciRS-7 in combination with CA125 and CEA produced a higher AUC value $(A U C=0.997)$ than any other
Table 2. Association of ciRS-7 expression with the clinical characteristics of cervical patients.

\begin{tabular}{|c|c|c|c|c|c|c|}
\hline \multirow{2}{*}{$\begin{array}{l}\text { Clinical features } \\
\mathrm{N}=352\end{array}$} & \multicolumn{4}{|c|}{ ciRS-7 expression } & \multirow{2}{*}{$\chi^{2}$} & \multirow{2}{*}{ p-value } \\
\hline & \multicolumn{2}{|r|}{ Low } & \multicolumn{2}{|c|}{ High } & & \\
\hline \multicolumn{7}{|l|}{ Age (years) } \\
\hline$\geq 50$ & 83 & $43.46 \%$ & 108 & $56.54 \%$ & & \\
\hline$<50$ & 63 & $39.13 \%$ & 98 & $60.87 \%$ & 0.673 & 0.412 \\
\hline \multicolumn{7}{|l|}{ Menopause } \\
\hline Yes & 87 & $43.28 \%$ & 114 & $56.72 \%$ & & \\
\hline No & 59 & $39.07 \%$ & 92 & $60.93 \%$ & 0.630 & 0.427 \\
\hline \multicolumn{7}{|l|}{ Histology } \\
\hline Squamous cell cancer & 116 & $43.28 \%$ & 152 & $56.72 \%$ & & \\
\hline Adenocarcinoma & 30 & $35.71 \%$ & 54 & $64.29 \%$ & 1.510 & 0.219 \\
\hline \multicolumn{7}{|l|}{ Tumor size $(\mathrm{cm})$} \\
\hline$\geq 4$ & 36 & $32.73 \%$ & 74 & $67.27 \%$ & & \\
\hline$<4$ & 110 & $45.45 \%$ & 132 & $54.55 \%$ & 5.047 & 0.025 \\
\hline \multicolumn{7}{|l|}{ Tumor differentiation } \\
\hline Poor & 38 & $36.19 \%$ & 67 & $63.81 \%$ & & \\
\hline Well-moderate & 108 & $43.72 \%$ & 139 & $56.28 \%$ & 1.723 & 0.189 \\
\hline \multicolumn{7}{|l|}{ FIGO stage } \\
\hline II & 43 & $33.86 \%$ & 84 & $66.14 \%$ & & \\
\hline I & 103 & $45.78 \%$ & 122 & $54.22 \%$ & 4.751 & 0.029 \\
\hline \multicolumn{7}{|l|}{ Depth of invasion } \\
\hline$\geq 2 / 3$ & 49 & $34.75 \%$ & 92 & $65.25 \%$ & & \\
\hline$<2 / 3$ & 97 & $45.97 \%$ & 114 & $54.03 \%$ & 4.383 & 0.036 \\
\hline \multicolumn{7}{|l|}{ Vascular involvement } \\
\hline Positive & 56 & $36.13 \%$ & 99 & $63.87 \%$ & & \\
\hline Negative & 90 & $45.69 \%$ & 107 & $54.31 \%$ & 3.264 & 0.071 \\
\hline \multicolumn{7}{|l|}{ Lymph nodes metastasis } \\
\hline Positive & 41 & $31.78 \%$ & 88 & $68.22 \%$ & & \\
\hline Negative & 105 & $47.09 \%$ & 118 & $52.91 \%$ & 7.884 & 0.005 \\
\hline \multicolumn{7}{|l|}{ HPV infection } \\
\hline Positive & 77 & $34.53 \%$ & 146 & $65.47 \%$ & & \\
\hline Negative & 69 & $79.38 \%$ & 60 & $46.51 \%$ & 12.10 & $<0.001$ \\
\hline
\end{tabular}


Table 3. Correlation between clinical features of cervical patients and their prognosis based on conduction of univariate and multivariate analyses.

\begin{tabular}{|c|c|c|c|c|c|c|}
\hline \multirow{2}{*}{ Clinical features } & \multicolumn{3}{|c|}{ Univariate analysis } & \multicolumn{3}{|c|}{ Multivariate analysis } \\
\hline & Hazard Ratio & $95 \% \mathrm{CI}$ & p-value & Hazard Ratio & $95 \% \mathrm{CI}$ & p-value \\
\hline \multicolumn{7}{|l|}{ ciRS-7 expression } \\
\hline High vs. Low & 0.49 & $0.31-0.79$ & 0.003 & 0.60 & $0.36-0.98$ & 0.041 \\
\hline \multicolumn{7}{|l|}{ Age (years) } \\
\hline$\geq 50$ vs. $<50$ & 1.13 & $0.72-1.75$ & 0.598 & 1.22 & $0.76-1.95$ & 0.413 \\
\hline \multicolumn{7}{|l|}{ Menopause } \\
\hline Yes vs. No & 1.29 & $0.82-2.02$ & 0.266 & 1.40 & $0.87-2.24$ & 0.164 \\
\hline \multicolumn{7}{|l|}{ Histology } \\
\hline Squamous cell cancer vs. Adenocarcinoma & 1.42 & $0.83-2.42$ & 0.201 & 1.49 & $0.84-2.64$ & 0.172 \\
\hline \multicolumn{7}{|l|}{ Tumor size $(\mathrm{cm})$} \\
\hline$\geq 4$ vs. $<4$ & 1.69 & $1.06-2.70$ & 0.027 & 1.48 & $0.90-2.45$ & 0.123 \\
\hline \multicolumn{7}{|l|}{ Tumor differentiation } \\
\hline Poor vs. Well-moderate & 0.94 & $0.58-1.52$ & 0.788 & 0.81 & $0.49-1.35$ & 0.421 \\
\hline \multicolumn{7}{|l|}{ FIGO stage } \\
\hline II vs. I & 1.94 & $1.23-3.06$ & 0.004 & 1.78 & $1.10-2.88$ & 0.018 \\
\hline \multicolumn{7}{|l|}{ Depth of invasion } \\
\hline$\geq 2 / 3$ vs. $<2 / 3$ & 1.64 & $1.05-2.57$ & 0.030 & 1.63 & $1.01-2.61$ & 0.044 \\
\hline \multicolumn{7}{|l|}{ Vascular involvement } \\
\hline Positive vs. Negative & 1.15 & $0.74-1.79$ & 0.539 & 1.06 & $0.66-1.70$ & 0.811 \\
\hline \multicolumn{7}{|l|}{ Lymph nodes metastasis } \\
\hline Positive vs. Negative & 1.43 & $0.91-2.25$ & 0.122 & 1.15 & $0.71-1.87$ & 0.563 \\
\hline \multicolumn{7}{|l|}{ HPV infection } \\
\hline Positive vs. Negative & 1.79 & $1.11-2.88$ & 0.017 & 1.58 & $0.95-2.62$ & 0.078 \\
\hline
\end{tabular}

Table 4. The efficacy of ciRS-7, CEA and CA125 in diagnosing cervical cancer.

\begin{tabular}{|c|c|c|c|c|c|c|}
\hline Biomarkers & Grouping & Value & Sensitivity & Specificity & AUC & $95 \% \mathrm{CI}$ \\
\hline \multirow[t]{3}{*}{ ciRS-7 } & Cervical cancer vs. Healthy control & 4.325 & 0.956 & 0.898 & 0.972 & $0.960-0.984$ \\
\hline & CIN vs. Healthy control & 2.840 & 0.700 & 0.902 & 0.879 & $0.848-0.910$ \\
\hline & Cervical cancer vs. CIN & 5.500 & 0.887 & 0.730 & 0.868 & $0.839-0.898$ \\
\hline \multirow[t]{3}{*}{ CEA } & Cervical cancer vs. Healthy control & 5.340 & 0.956 & 0.767 & 0.885 & $0.857-0.912$ \\
\hline & CIN vs. Healthy control & 4.590 & 0.872 & 0.809 & 0.920 & $0.895-0.944$ \\
\hline & Cervical cancer vs. CIN & 7.985 & 0.966 & 0.582 & 0.734 & $0.692-0.776$ \\
\hline \multirow[t]{3}{*}{ CA125 } & Cervical cancer vs. Healthy control & 35.065 & 0.974 & 0.741 & 0.881 & $0.852-0.910$ \\
\hline & CIN vs. Healthy control & 29.015 & 0.872 & 0.554 & 0.739 & $0.690-0.788$ \\
\hline & Cervical cancer vs. CIN & 47.500 & 0.828 & 0.540 & 0.730 & $0.689-0.772$ \\
\hline
\end{tabular}

AUC: area under the receiver operating curve; 95\%CI: 95\% confidence interval.

Table 5. The combination of ciRS7, CEA and CA125 for diagnosing cervical cancer.

\begin{tabular}{|c|c|c|c|c|c|}
\hline Biomarkers & Grouping & Sensitivity & Specificity & AUC & $95 \% \mathrm{CI}$ \\
\hline \multirow[t]{3}{*}{ ciRS7-CEA } & Cervical cancer vs. Healthy control & 0.987 & 0.952 & 0.990 & $0.984-0.997$ \\
\hline & CIN vs. Healthy control & 0.885 & 0.961 & 0.973 & $0.960-0.986$ \\
\hline & Cervical cancer vs. CIN & 0.951 & 0.773 & 0.904 & $0.879-0.929$ \\
\hline \multirow[t]{3}{*}{ ciRS7-CA125 } & Cervical cancer vs. Healthy control & 0.974 & 0.949 & 0.990 & $0.983-0.996$ \\
\hline & CIN vs. Healthy control & 0.797 & 0.858 & 0.912 & $0.886-0.938$ \\
\hline & Cervical cancer vs. CIN & 0.892 & 0.787 & 0.895 & $0.869-0.920$ \\
\hline \multirow[t]{3}{*}{ CEA-CA125 } & Cervical cancer vs. Healthy control & 0.965 & 0.889 & 0.963 & $0.948-0.979$ \\
\hline & CIN vs. Healthy control & 0.819 & 0.936 & 0.946 & $0.927-0.965$ \\
\hline & Cervical cancer vs. CIN & 0.966 & 0.582 & 0.814 & $0.779-0.848$ \\
\hline \multirow[t]{3}{*}{ ciRS7-CEA-CA125 } & Cervical cancer vs. Healthy control & 0.996 & 0.963 & 0.997 & $0.994-1.000$ \\
\hline & CIN vs. Healthy control & 0.938 & 0.941 & 0.980 & $0.969-0.991$ \\
\hline & Cervical cancer vs. CIN & 0.946 & 0.804 & 0.921 & $0.899-0.943$ \\
\hline
\end{tabular}

AUC: area under the receiver operating curve; 95\%CI: 95\% confidence interval. 
diagnostic strategy that was made up of two biomarkers (Table 5, Figure 2D).

Contribution of ciRS-7 to altering the viability, proliferation and apoptosis of CC cell lines. The expression of ciRS-7 was significantly upregulated within Hela, CaSki, C33A and $\mathrm{SiHa}$ cell lines, when compared with ESC cell line $(\mathrm{p}<0.05)$ (Figure $3 \mathrm{~A}$ ). Concurrently, expressions of pro-proliferation molecules, including Ki-67, PCNA and cyclin D1, were raised within the 4 CC cell lines, as compared with ESC cell line $(\mathrm{p}<0.05)$. Interestingly, the changing tendency of ciRS-7 expression was consistent with that of Ki-67, PCNA and cyclin D1 expressions among the CC cell lines (Figure 3B). Besides, CC cell lines were in possession of lower E-cadherin expression and higher $\mathrm{N}$-cadherin/vimentin expression than ESC cell line $(\mathrm{p}<0.05)$, and expressions of E-cadherin,
$\mathrm{N}$-cadherin and vimentin were altered more observably within Hela/C33A cell lines than within CaSki/SiHa cell lines (Figure 3C).

Since the ciRS-7 expression was upregulated more significantly in Hela and C33A cell lines than in any other cell line, the two cell lines were arranged for subsequent cellular experiments. After transfection of pcDNA3.1-ciRS-7, the expression of ciRS-7 was obviously elevated in both Hela and C33A cell lines $(\mathrm{p}<0.05)$ (Figure $4 \mathrm{~A})$. Moreover, transfection of pcDNA3.1-ciRS-7 seemed to bestow Hela and C33A cells with strengthened viability (Figure 4B) and proliferative ability (Figure 4C), and apoptosis (Figure 4D) of the CC cells was hindered drastically after exposure to pcDNA3.1-ciRS-7 $(\mathrm{p}<0.05)$. Not only that, CC cells transfected with pcDNA3.1ciRS-7 also presented upregulated expressions of Ki-67,
A Cervival cancer vs. Healthy control
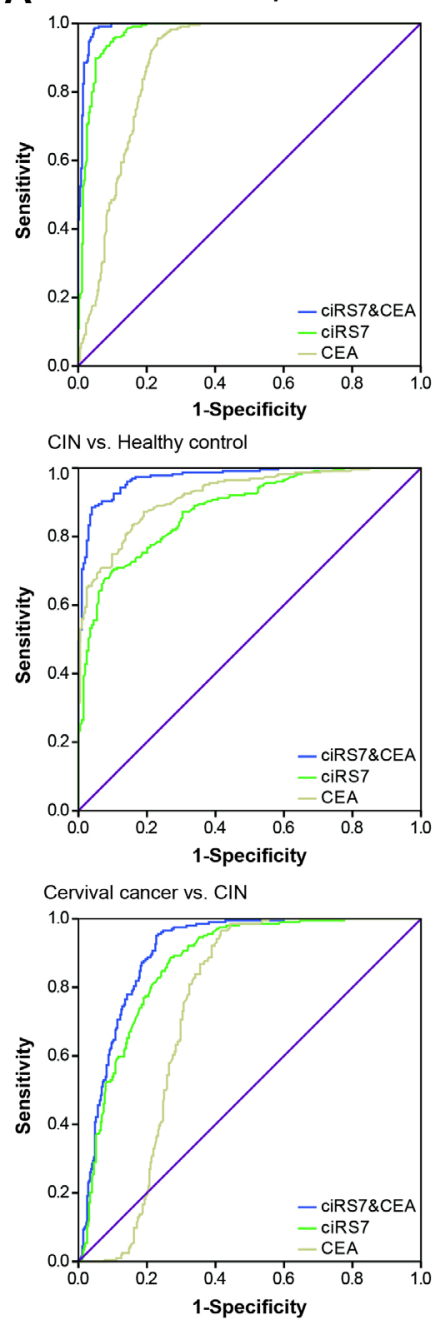

B Cervival cancer vs. Healthy control
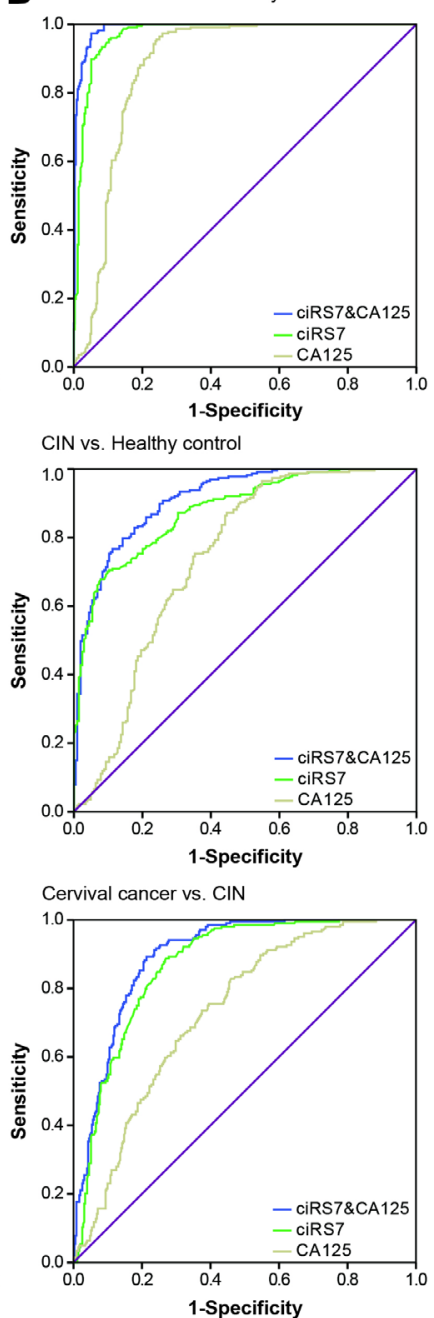

C Cervival cancer vs. Healthy control
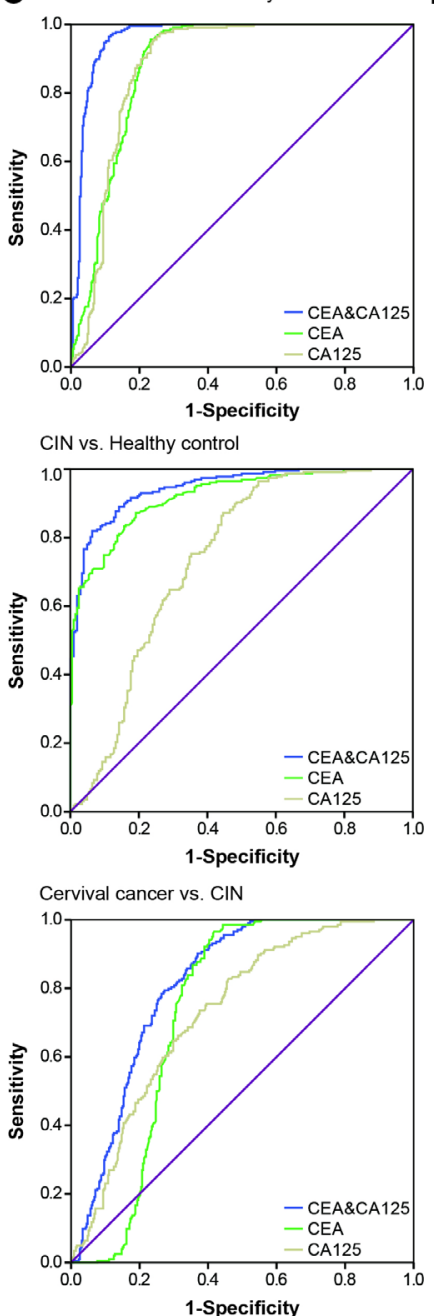

D Cervival cancer vs. Healthy control

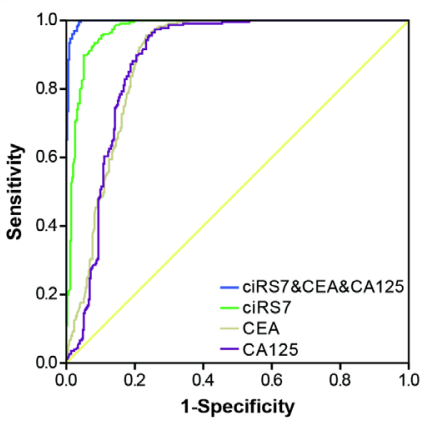

CIN vs. Healthy control
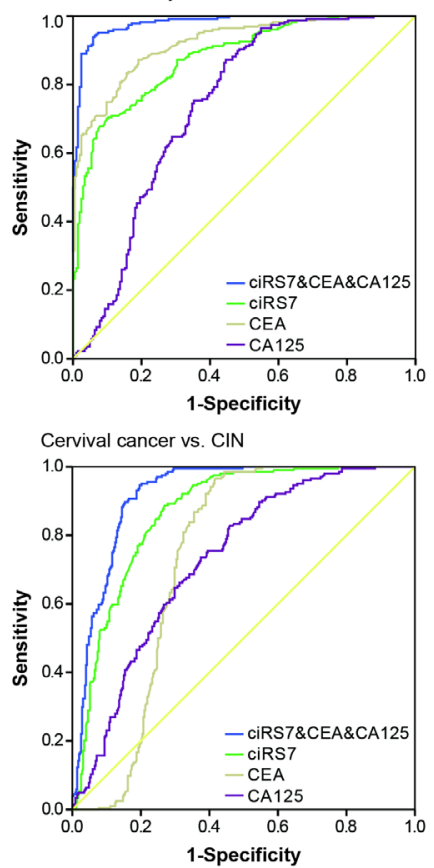

Figure 2 The diagnostic performance of ciRS-7, CEA and CA125 in cervical cancer and CIN. A) The synergic role of ciRS-7 and CEA were evaluated concerning differentiation of cervical cancer and CIN from healthy controls. B) ciRS-7 and CA125 were managed to collaboratively diagnose cervical cancer and CIN. C) The couple of CEA and CA125 were apprised in terms of their diagnostic potency for cervical cancer and CIN. D) The synthetic function of ciRS-7, CEA and CA125 was evaluated in diagnosis of cervical cancer and CIN. 


\section{A}

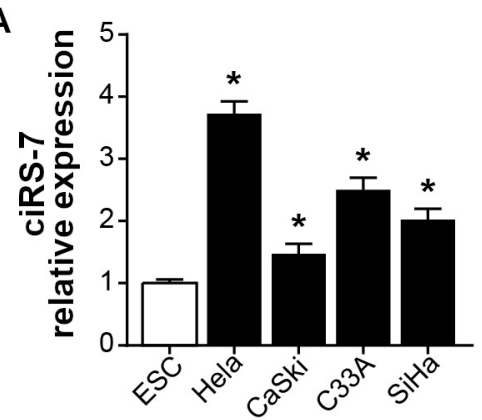

B

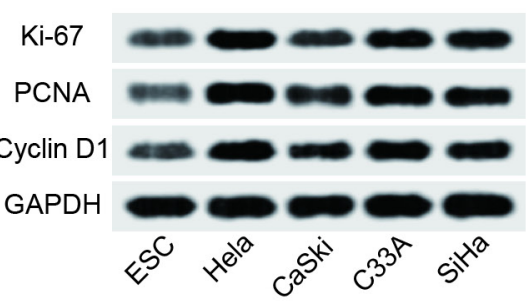

C

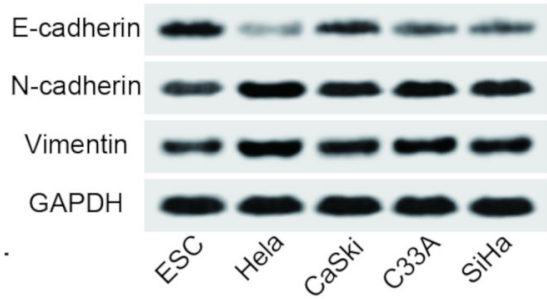

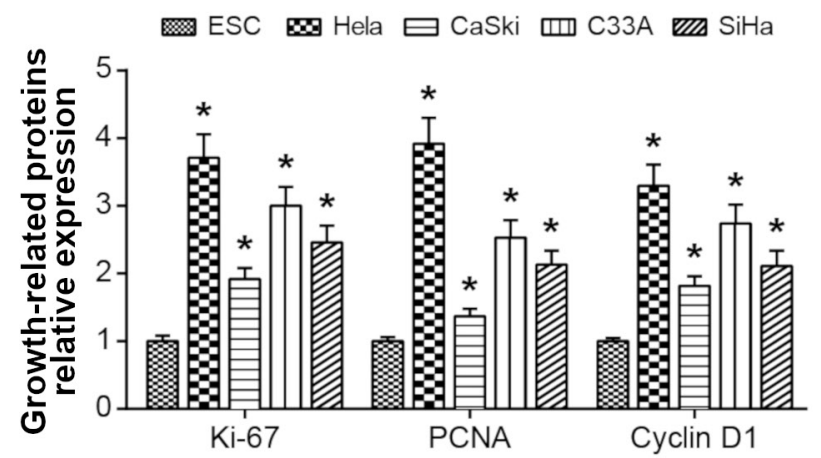

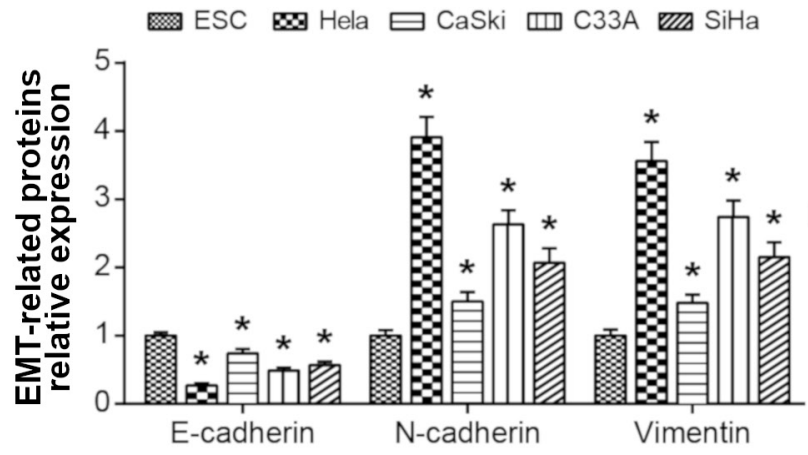

Figure 3. The expression of ciRS-7, growth- and EMT-related proteins within cervical cancer cells. A) The expression of ciRS-7 was determined within ESC, Hela, CaSki, C33A and SiHa cell lines. ${ }^{*}$ : $p<0.05$ when compared with ESC. B) Expressions of Ki-67, PCNA and cyclin D1 were compared among ESC, CaSki, Hela, C33A and SiHa cell lines. ${ }^{*}:$ p $<0.05$ when compared with ESC. C) The molecules specific to epithelial-mesenchymal transition were evaluated within ESC, CaSki, Hela, C33A and SiHa cell lines. ${ }^{*}:$ p $<0.05$ when compared with ESC.

PCNA and cyclinD1, which were proteins representative of intensified cell proliferation $(\mathrm{p}<0.05)$ (Figure 4E).

The role of ciRS-7 in modulating EMT process of CC cell lines. Elevating the expression of ciRS-7 could reinforce the invasive ability of Hela and C33A cell lines, when compared with pcDNA3.1 group $(\mathrm{p}<0.05)$ (Figure $5 \mathrm{~A})$. Abiding by a similar trend, the migratory capacity of Hela and $\mathrm{C} 33 \mathrm{~A}$ cell lines was also facilitated after transfection of pcDNA3.1-ciRS-7 $(\mathrm{p}<0.05)$ (Figure 5B). In the meantime, elevated expressions of $\mathrm{N}$-cadherin and vimentin, along with decreased E-cadherin expression, were detected within CC cells that were treated by pcDNA3.1-ciRS-7, as compared to untreated cells $(\mathrm{p}<0.05)$ (Figure 5C).

\section{Discussion}

Currently, the definite diagnoses of CC were mainly dependent on cervical scraping smear and colposcope, which failed to ensure accurate diagnosis of all CC cases due to obscure symptoms present in early-stage CC. Notably, tumor biomarkers within blood already assumed concentration discrepancies at the early stage of tumorigenesis [19], suggesting the practicability of applying specific biomarkers for diagnosis of early-stage neoplasms. Besides, the CC patients that have progressed into the advanced stage were usually tracked with a relatively short life span, although certain treatment strategies, such as surgery and radiotherapy, were broadly available. Thus, seeking for sensitive biomarkers for early stage CC might be beneficial to efficaciously diagnosing and treating CC.

Notably, the expressional fashion of circRNAs usually varied with pathological changes of human bodies, providing a direction that circRNAs might sensitively indicate the deterioration of neoplasms [20]. For instance, hsa_circ_000284 and hsa_circ_0018289 were both differentially expressed during exacerbation of CC $[21,22]$. Within this investigation, ciRS-7 was implied as an oncogene for CC, and overexpression of ciRS-7 within CC tissues could symbolize unfavorable prognosis of CC patients (Figure 1C). Besides the clinical evidences, the in vitro experiments also demonstrated that upregulation of ciRS-7 expression tended to accelerate proliferation and EMT process of CC cells (Figure 3-5), which agreed with other tumors [11, 12]. In fact, former studies have proposed hidden linkages of ciRS-7 with CC progression. For instance, signaling pathways that were modulated by ciRS-7 were crucial involvers underlying CC pathogenesis, such as PI3K/AKT signaling [11, 23] and NF-kB signaling $[24,25]$. The PI3K/AKT signaling therein was able to enhance both chemo- and radio- resistances of CC cell lines $[26,27]$, in addition to intensifying proliferation, migration and invasion of CC cells [28]. As for NF-kB signaling, its restraining production of cleaved caspase- 3 
A

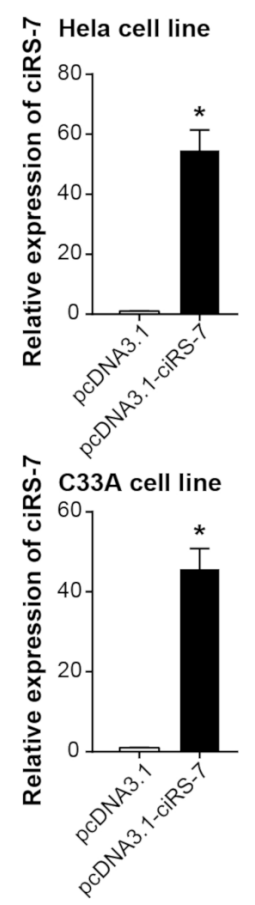

B

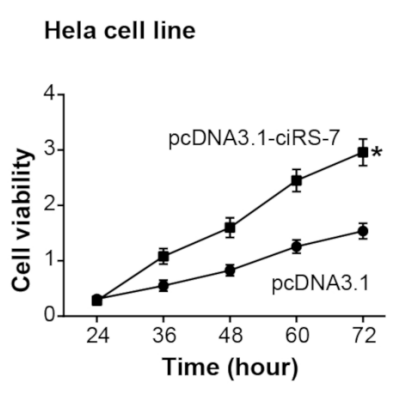

C33A cell line

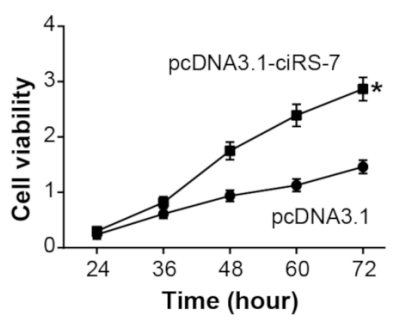

C
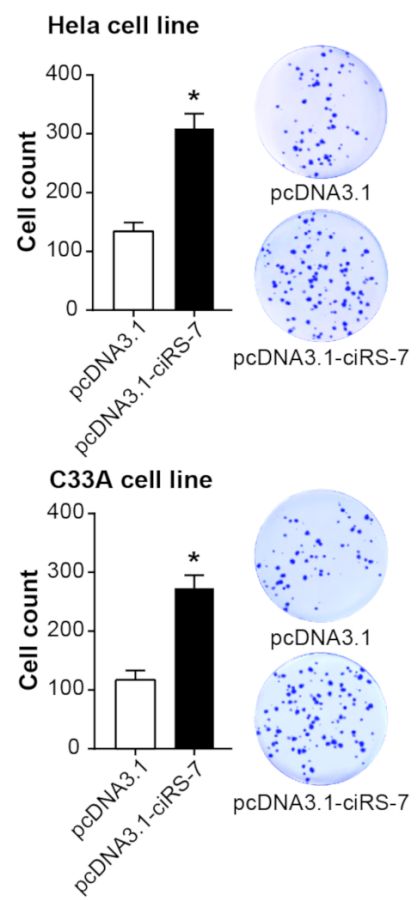

D
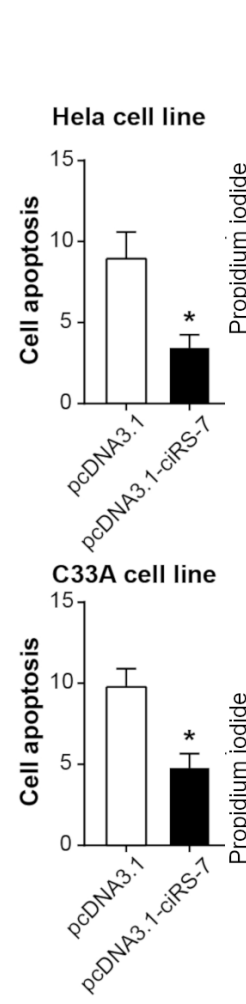

pcDNA3.1

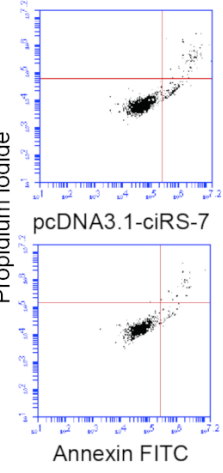

pcDNA3.1

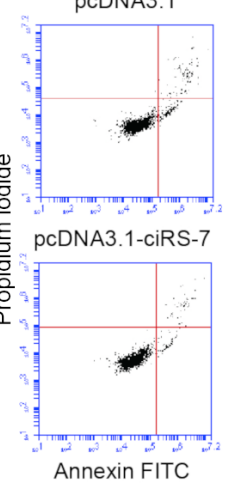

E

Hela cell line

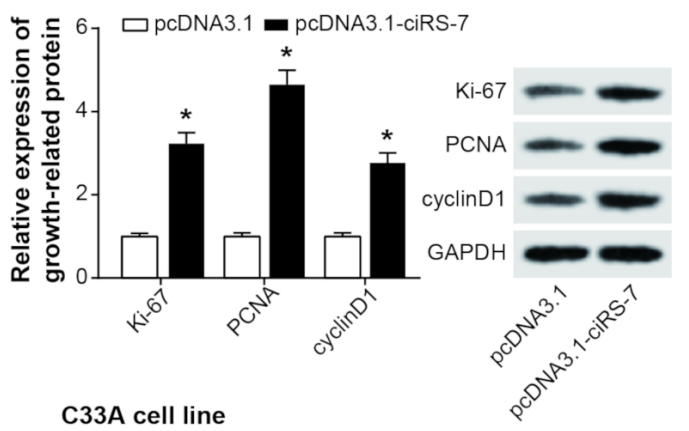

C33A cell line
$\square$ pcDNA3.1 $\square$ pcDNA3.1-ciRS-7
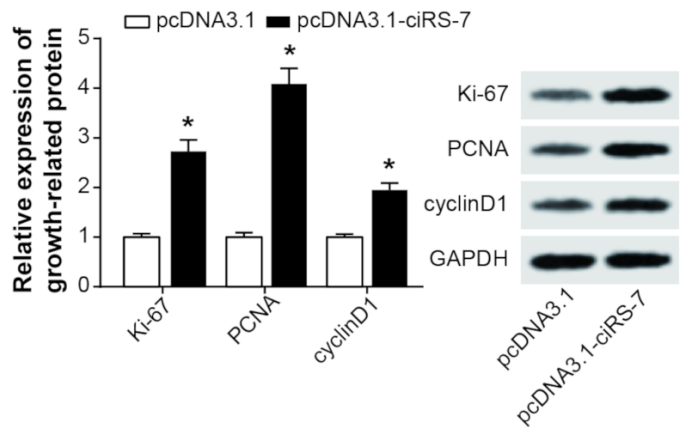

Figure 4. The effect of ciRS-7 on the viability, proliferation and apoptosis of cervical cancer cells. A) The ciRS-7 expression was assessed after respective transfection of pcDNA3.1-ciRS-7 and pcDNA3.1. ${ }^{\star}: p<0.05$ when compared with pcDNA3.1 group. B) The viability of Hela and C33A cell lines was appraised in the pcDNA3.1-ciRS-7 and pcDNA3.1 groups. ${ }^{*}: \mathrm{p}<0.05$ when compared with pcDNA3.1 group. C) The proliferative ability of Hela and C33A cell lines was detected in the pcDNA3.1-ciRS-7 and pcDNA3.1 groups. ${ }^{*}: \mathbf{p}<0.05$ when compared with pcDNA3.1 group. D) The apoptotic condition of Hela and C33A cell lines was contrasted between pcDNA3.1-ciRS-7 and pcDNA3.1 groups. ${ }^{*}$ : $p<0.05$ when compared with pcDNA3.1 group. E) The expressions of Ki-67, PCNA and cyclinD1 were determined within Hela and C33A cell lines in the pcDNA3.1-ciRS-7 and pcDNA3.1 groups. ${ }^{*}: \mathrm{p}<0.05$ when compared with pcDNA3.1 group. 
A

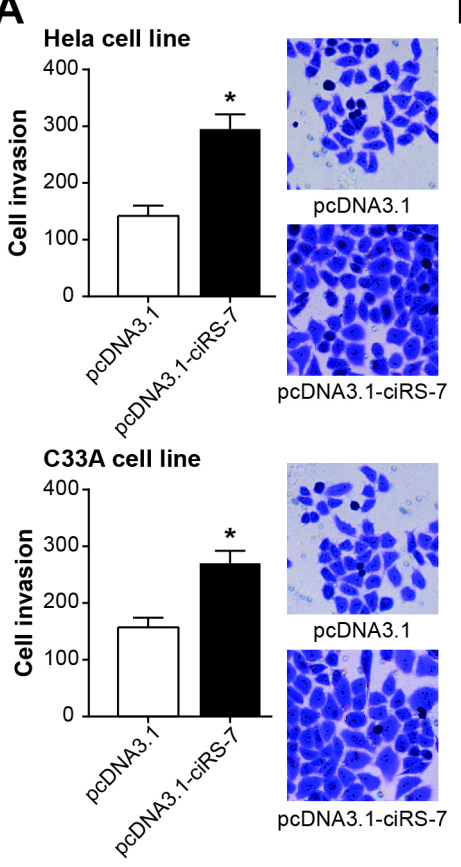

B

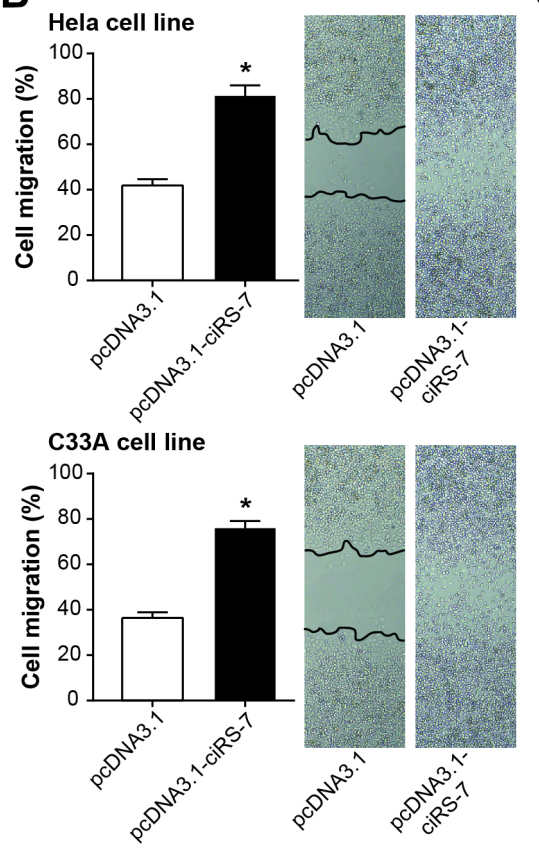

C

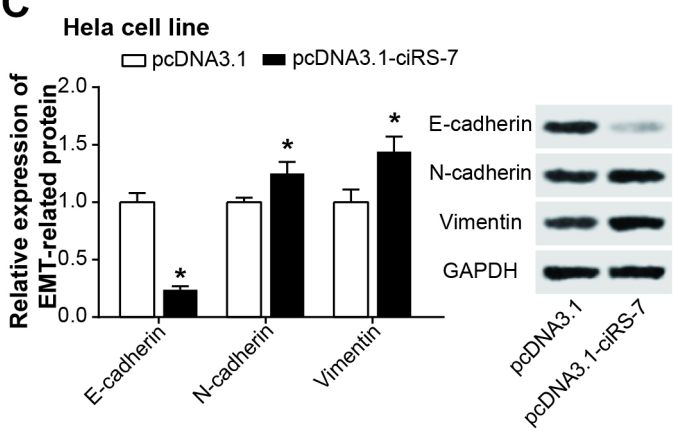

C33A cell line

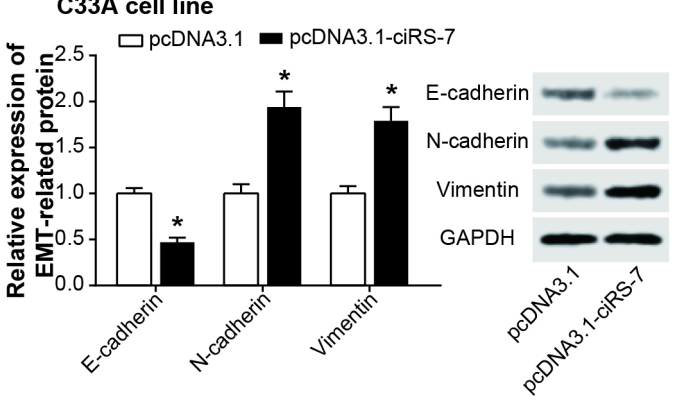

Figure 5 The role of ciRS-7 in modulating invasion (A), migration (B) and expression of proteins specific to epithelial-mesenchymal transition (EMT) (C) of Hela and C33A cell lines. ${ }^{\star}$ : $\mathrm{p}<0.05$ when compared with pcDNA3.1 group.

could curb apoptosis of CC cells [29], which might explain why activation of NF-kB signaling was associated with unfavorable prognosis of CC patients [25]. In addition, there were massive potential for ciRS-7 to form circRNA-miRNA networks so as to trigger CC onset [11], although miRNAs that possessed ciRS-7-binding sites were largely untapped [30]. Of course, detailed researches were demanded to validate direct relations between ciRS-7 and the abovementioned miRNAs or signaling pathways underlying CC etiology.

Inspired by the significance of ciRS-7 in regulating CC etiology, we further observed that ciRS-7 was outstanding in differentiating CC patients from CIN patients and healthy controls (Figure 2). In fact, circRNAs, characterized by shortage of 5'- and 3'- ends, could avoid being digested by exonuclease and thereby maintain their original high-level conservation, which might aid to enhance the sensitivity of ciRS-7 for CC diagnosis. Nevertheless, since that ciRS-7 also participated in the pathogenesis of disorders other than CC, including acute myocardial infarction [30], systemic lupus erythematosus [31], hepatocellular carcinoma [32], biliary duct cancer [33] and colorectal cancer [34], the specificity of ciRS-7 for CC diagnosis was confined (Table 4). To improve this aspect, further elucidation of CC-specific biomarkers that were modulated by ciRS-7 might be of assistance.

Furthermore, to highlight the diagnostic performance of ciRS-7, CEA and CA125 were chosen as the control biomarkers (Figure 2, Tables 4-5). The CA125 was a common marker for CC, and its high level assumed intimate associations with enlarged tumor size, advanced stage and aggressive lymphatic metastasis of CC patients [31, 32]. Regarding CEA, its level was raised with neoplastic metastasis and recurrence, and dynamically observing its serum level could help to conjecture prognosis of CC patients [33]. However, the CEA and CA125 might not be appropriate for specific screening, allowing for that they displayed expressional variations in a wide range of tumors [34, 35]. Interestingly, the combined capacity of ciRS-7, CEA and CA125 in diagnosing $\mathrm{CC}$ was stronger than that of any single molecule (Figure 2D, Table 5), which might be attributed to that single biomarkers could merely participate in a small portion of CC etiology. Thus, screening a group of desired biomarkers for combined diagnosis of CC might be more clinically valuable, because multiple biomarkers could perceive the physiological changes of CC more sensitively.

Conclusively, ciRS-7 was a promising biomarker for diagnosis and even treatment of $\mathrm{CC}$, and its combination with CEA and CA125 were recommended for diagnosis of CC. Nevertheless, miRNAs specifically regulated by ciRS-7 in CC were unexplored within this study, and their combination with ciRS-7 could pronouncedly improve the specificity in CC diagnosis. Furthermore, the sample size of this investigation was relatively small, which might generate biased results. Besides, merely a Chinese cohort was focused on here, whether the results of this study could be generalized to other ethnicities remained doubtful. Finally, though 
ciRS-7 expression displayed some connection with the status of $\mathrm{HPV}$, the reasons explaining this correlation remained unclear. Above all, the questions raised as above should be carefully settled in future.

\section{References}

[1] TORRE LA, BRAY F, SIEGEL RL, FERLAY J, LORTETTIEULENT J et al. Global cancer statistics, 2012. CA Cancer J Clin 2015; 65: 87-108. https://doi.org/10.3322/caac.21262

[2] HU SY, ZHENG RS, ZHAO FH, ZHANG SW, CHEN WQ et al. [Trend analysis of cervical cancer incidence and mortality rates in Chinese women during 1989-2008]. Zhongguo Yi Xue Ke Xue Yuan Xue Bao. 2014; 36: 119-125. https://doi. org/10.3881/j.issn.1000-503X.2014.02.001

[3] LIU L, YU X, GUO X, TIAN Z, SU M et al. miR-143 is downregulated in cervical cancer and promotes apoptosis and inhibits tumor formation by targeting Bcl-2. Mol Med Rep 2012; 5: 753-760. https://doi.org/10.3892/mmr.2011.696

[4] RUDOLF E, RUDOLF K, CERVINKA M. Selenium activates p53 and p38 pathways and induces caspase-independent cell death in cervical cancer cells. Cell Biol Toxicol 2008; 24: 123141. https://doi.org/10.1007/s10565-007-9022-1

[5] YUAN Y, ZHANG J, CAI L, DING C, WANG X et al. Leptin induces cell proliferation and reduces cell apoptosis by activating c-myc in cervical cancer. Oncol Rep 2013; 29: 22912296. https://doi.org/10.3892/or.2013.2390

[6] SANGER HL, KLOTZ G, RIESNER D, GROSS HJ, KLEINSCHMIDT AK. Viroids are single-stranded covalently closed circular RNA molecules existing as highly base-paired rod-like structures. Proc Natl Acad Sci U S A 1976; 73: 38523856. https://doi.org/10.1073/pnas.73.11.3852

[7] KOLAKOFSKY D. Isolation and characterization of Sendai virus DI-RNAs. Cell 1976; 8: 547-555. https://doi. org/10.1016/0092-8674(76)90223-3

[8] ROBERTS MJ, RICHARDS RS, GARDINER RA, SELTH LA. Seminal fluid: a useful source of prostate cancer biomarkers? Biomark Med 2015; 9: 77-80. https://doi.org/10.2217/ bmm. 14.110

[9] WANG X, ZHANG Y, HUANG L, ZHANG J, PAN F et al. Decreased expression of hsa_circ_001988 in colorectal cancer and its clinical significances. Int J Clin Exp Pathol 2015; 8: 16020-16025.

[10] XU L, ZHANG M, ZHENG X, YI P, LAN C et al. The circular RNA ciRS-7 (Cdrlas) acts as a risk factor of hepatic microvascular invasion in hepatocellular carcinoma. J Cancer Res Clin Oncol 2017; 143: 17-27. https://doi.org/10.1007/ s00432-016-2256-7

[11] PAN H, LI T, JIANG Y, PAN C, DING Y et al. Overexpression of Circular RNA ciRS-7 Abrogates the Tumor Suppressive Effect of miR-7 on Gastric Cancer via PTEN/PI3K/ AKT Signaling Pathway. J Cell Biochem 2018; 119: 440-446. https://doi.org/10.1002/jcb.26201

[12] LI RC, KE S, MENG FK, LU J, ZOU XJ et al. CiRS-7 promotes growth and metastasis of esophageal squamous cell carcinoma via regulation of miR-7/HOXB13. Cell Death Dis 2018; 9: 838. https://doi.org/10.1038/s41419-018-0852-y
[13] HANSEN TB, JENSEN TI, CLAUSEN BH, BRAMSEN JB, FINSEN B et al. Natural RNA circles function as efficient microRNA sponges. Nature 2013; 495: 384-388. https://doi. org/10.1038/nature11993

[14] LIU S, ZHANG P, CHEN Z, LIU M, LI X et al. MicroRNA-7 downregulates XIAP expression to suppress cell growth and promote apoptosis in cervical cancer cells. FEBS Lett 2013; 587: 2247-2253. https://doi.org/10.1016/j.febslet.2013.05.054

[15] GONG Y, MAO J, WU D, WANG X, LI L et al. Circ-ZEB1.33 promotes the proliferation of human HCC by sponging miR200a-3p and upregulating CDK6. Cancer Cell Int 2018; 18: 116. https://doi.org/10.1186/s12935-018-0602-3

[16] MEMCZAK S, JENS M, ELEFSINIOTI A, TORTI F, KRUEGER J et al. Circular RNAs are a large class of animal RNAs with regulatory potency. Nature 2013; 495: 333-338. https://doi.org/10.1038/nature11928

[17] SHI Y, LUO X, LI P, TAN J, WANG X et al. miR-7-5p suppresses cell proliferation and induces apoptosis of breast cancer cells mainly by targeting REGgamma. Cancer Lett 2015; 358: 27-36. https://doi.org/10.1016/j.canlet.2014.12.014

[18] LIU H, HUANG J, PENG J, WU X, ZHANG Y et al. Upregulation of the inwardly rectifying potassium channel Kir2.1 (KCNJ2) modulates multidrug resistance of small-cell lung cancer under the regulation of miR-7 and the Ras/MAPK pathway. Mol Cancer 2015; 14: 59. https://doi.org/10.1186/ s12943-015-0298-0

[19] MOLINA R, FILELLA X, AUGE JM, FUENTES R, BOVER I et al. Tumor markers (CEA, CA 125, CYFRA 21-1, SCC and NSE) in patients with non-small cell lung cancer as an aid in histological diagnosis and prognosis. Comparison with the main clinical and pathological prognostic factors. Tumour Biol 2003; 24: 209-218. https://doi.org/10.1159/000074432

[20] MENG S, ZHOU H, FENG Z, XU Z, TANG Y et al. CircRNA: functions and properties of a novel potential biomarker for cancer. Mol Cancer 2017; 16: 94. https://doi. org/10.1186/s12943-017-0663-2

[21] MA HB, YAO YN, YU JJ, CHEN XX, LI HF. Extensive profiling of circular RNAs and the potential regulatory role of circRNA-000284 in cell proliferation and invasion of cervical cancer via sponging miR-506. Am J Transl Res 2018; 10: 592-604.

[22] GAO YL, ZHANG MY, XU B, HAN LJ, LAN SF et al. Circular RNA expression profiles reveal that hsa_circ_0018289 is up-regulated in cervical cancer and promotes the tumorigenesis. Oncotarget 2017; 8: 86625-86633. https://doi. org/10.18632/oncotarget.21257

[23] LI L, SUN JX, WANG XQ, LIU XK, CHEN XX et al. Notoginsenoside R7 suppresses cervical cancer via PI3K/PTEN/ Akt/mTOR signaling. Oncotarget 2017; 8: 109487-109496. https://doi.org/10.18632/oncotarget.22721

[24] HUANG H, WEI L, QIN T, YANG N, LI Z et al. Circular RNA ciRS-7 triggers the migration and invasion of esophageal squamous cell carcinoma via miR-7/KLF4 and NF-kappaB signals. Cancer Biol Ther 2019; 20: 73-80. https://doi.or g/10.1080/15384047.2018.1507254 
[25] LI J, JIA H, XIE L, WANG X, WANG X et al. Association of constitutive nuclear factor-kappaB activation with aggressive aspects and poor prognosis in cervical cancer. Int J Gynecol Cancer 2009; 19: 1421-1426. https://doi.org/10.1111/ IGC.0b013e3181b70445

[26] XIA S, ZHAO Y, YU S, ZHANG M. Activated PI3K/Akt/ COX-2 pathway induces resistance to radiation in human cervical cancer HeLa cells. Cancer Biother Radiopharm 2010; 25: 317-323. https://doi.org/10.1089/cbr.2009.0707

[27] DU J, WANG L, LI C, YANG H, LI Y et al. MicroRNA-221 targets PTEN to reduce the sensitivity of cervical cancer cells to gefitinib through the PI3K/Akt signaling pathway. Tumour Biol 2016; 37: 3939-3947. https://doi.org/10.1007/ s13277-015-4247-8

[28] ZHANG D, SUN G, ZHANG H, TIAN J, LI Y. Long noncoding RNA ANRIL indicates a poor prognosis of cervical cancer and promotes carcinogenesis via PI3K/Akt pathways. Biomed Pharmacother 2017; 85: 511-516. https://doi. org/10.1016/j.biopha.2016.11.058

[29] DANG YP, YUAN XY, TIAN R, LI DG, LIU W. Curcumin improves the paclitaxel-induced apoptosis of HPV-positive human cervical cancer cells via the NF-kappaB-p53-caspase-3 pathway. Exp Ther Med 2015; 9: 1470-1476. https:// doi.org/10.3892/etm.2015.2240

[30] SANG M, MENG L, SANG Y, LIU S, DING P et al. Circular RNA ciRS-7 accelerates ESCC progression through acting as a miR-876-5p sponge to enhance MAGE-A family expression. Cancer Lett 2018; 426: 37-46. https://doi.org/10.1016/j. canlet.2018.03.049
[31] PORIKA M, VEMUNOORI AK, TIPPANI R, MOHAMMAD A, BOLLAM SR et al. Squamous cell carcinoma antigen and cancer antigen 125 in southern Indian cervical cancer patients. Asian Pac J Cancer Prev 2010; 11: 17451747.

[32] TSAI CC, LIU YS, HUANG EY, HUANG SC, CHANG HW et al. Value of preoperative serum CA125 in early-stage adenocarcinoma of the uterine cervix without pelvic lymph node metastasis. Gynecol Oncol 2006; 100: 591-595. https:// doi.org/10.1016/j.ygyno.2005.09.049

[33] YOON SM, SHIN KH, KIM JY, SEO SS, PARK SY et al. The clinical values of squamous cell carcinoma antigen and carcinoembryonic antigen in patients with cervical cancer treated with concurrent chemoradiotherapy. Int J Gynecol Cancer 2007; 17: 872-878. https://doi.org/10.1111/j.15251438.2007.00878.x

[34] LOCKER GY, HAMILTON S, HARRIS J, JESSUP JM, KEMENY N et al. ASCO 2006 update of recommendations for the use of tumor markers in gastrointestinal cancer. J Clin Oncol 2006; 24: 5313-5327. https://doi.org/10.1200/ JCO.2006.08.2644

[35] DUFFY MJ, VAN DALEN A, HAGLUND C, HANSSON L, HOLINSKI-FEDER E et al. Tumour markers in colorectal cancer: European Group on Tumour Markers (EGTM) guidelines for clinical use. Eur J Cancer 2007; 43: 1348-1360. https://doi.org/10.1016/j.ejca.2007.03.021 